\title{
Organic solar cells as high-speed data detectors for visible light communication: supplementary material
}

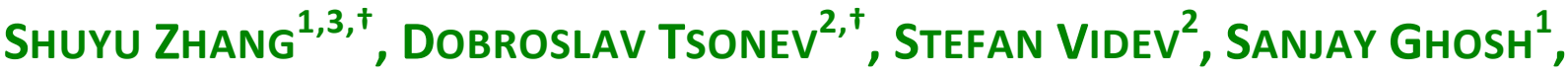 \\ Graham A. TURNBUlL ${ }^{1, *}$, IfOR D.W. SAMUEL ${ }^{1,{ }^{*}}$ ANd Harald HaAs ${ }^{2, *}$ \\ ${ }^{1}$ Organic Semiconductor Centre, SUPA, School of Physics and Astronomy, University of St Andrews, St Andrews, Fife KY16 9SS, UK \\ ${ }^{2}$ Institute for Digital Communications, Joint Research Institute for Signal and Image Processing, University of Edinburgh, Edinburgh EH9 3JL,UK \\ ${ }^{3}$ School of Information Science and Technology, Fudan University, Shanghai, 200433, China PR \\ *Corresponding authors: gat@st-andrews.ac.uk; idws@st-andrews.ac.uk; h.haas@ed.ac.uk
}

Published 24 June 2015

This document provides supplementary information to "Organic solar cells as high-speed data detectors for visible light communication," http://dx.doi.org/10.1364/optica.2.000607. Practical implementations of visible light communications (VLC) are documented here. (C) 2015 Optical Society of America

http://dx.doi.org/10.1364/optica.2.000607.s001

Practical implementations of VLC are realised using intensity modulation and directed detection (IM/DD). Therefore, a modulation signal has to be both real and non-negative. Because conventional orthogonal frequency division multiplexing (OFDM) generates a bipolar complex signal, Hermitian symmetry has to be imposed in the inverse fast Fourier transform (IFFT) during the signal generation process $[1,2]$. In OFDM, a total of $N_{\mathrm{fft}}$ subcarriers are equally distributed within the bandwidth of $\left[-T_{\mathrm{s}} / 2 ; T_{\mathrm{s}} / 2\right]$ where $T_{\mathrm{s}}$ is the time-domain sampling period of the communication system. Some subcarriers can be left unmodulated in order to avoid interference or because of insufficient signal-tonoise ratio (SNR) levels in the corresponding frequency band. The average spectral efficiency of the modulation scheme can be calculated as:

$$
\eta=\sum_{\substack{k=1 \\ M_{k}>0}}^{\frac{N_{\mathrm{ftt}}}{2}-1} \frac{\log _{2} M_{k}}{N_{\mathrm{fft}}+N_{\mathrm{cp}}}\left[\frac{\mathrm{bit}}{\mathrm{s} \cdot \mathrm{Hz}}\right],
$$

where $N_{\text {fft }}$ corresponds to the fast Fourier transform (FFT) size, Mk is the size of the $M$-ary quadrature amplitude modulation $(M$ QAM) constellation on subcarrier $\mathrm{k}, N_{\mathrm{cp}}$ is the OFDM cyclic prefix length. The single-sided bandwidth of the communication system is:

$$
B=\frac{1}{T_{\mathrm{S}}}[\mathrm{Hz}] .
$$

Using the bandwidth and the spectral efficiency, the data-rate of the system is:

$$
D=2 B \eta .
$$

In this work, the FFT size has been set to $N_{\mathrm{fft}}=1024$, and the cyclic prefix length has been set to $N_{\mathrm{cp}}=10$.

In order to accommodate the bipolar OFDM signal within the active region of the laser diode (LD), a suitable direct current bias is applied. It sets a positive operating point for the device around which the bipolar signal can be realised $[1,2]$. This modulation technique is referred to as direct-current-biased optical OFDM (DCO-OFDM). A typical time-domain OFDM signal is characterised by a high PAPR, which increases with the number of utilised subcarriers. The linear dynamic range of the LD is limited from below and from above due to the opto-electrical properties of the devices. As a result, the OFDM signal is clipped on both sides of its time-domain distribution. Simulation work and exhaustive experiments have shown that clipping levels at $-3.2 \sigma$ and $3.2 \sigma$, where $\sigma$ is the standard deviation of the time-domain signal, are approximately optimal in the digital signal conditioning step. The standard deviation of the OFDM signal is:

$$
\sigma^{2}=\sum_{k=1}^{\frac{N_{\mathrm{ftt}}}{2}-1} \frac{E_{\mathrm{b} k} \log _{2} M_{k}}{N_{\mathrm{fft}}},
$$

where $E_{\mathrm{b} k}$ is the energy per bit on subcarrier $k$. The pulse shaping and matched filtering operations are performed with a rootraised-cosine (RRC) filter with a roll-off factor of 0.1 and an oversampling ratio of 4 . The sampling frequency of the arbitrary waveform generator (AWG) is fixed at $F_{\mathrm{s}}=80 \mathrm{Ms} / \mathrm{s}$, which results in a maximum achievable single-sided bandwidth of $B=10 \mathrm{MHz}$. 
Channel estimation is performed with a pilot sequence that consists of random 4-QAM symbols with a uniform energy profile.

\section{References}

1. H. Elgala, R. Mesleh and H. Haas, IEEE Commun. Mag. 49, 56-62 (2011).

2. A. M. Khalid, G. Cossu, R. Corsini, P. Choudhury and E. Ciaramella, IEEE Photon. J. 4, 1465-1473 (2012). 